\title{
A case of tactile agnosia with a lesion restricted to the post- central gyrus
}

\author{
Bruno Estañol, José Fidel Baizabal-Carvallo, Horacio Sentíes-Madrid \\ Department of Neurology and Psychiatry, The National Institute of Medical Sciences and Nutrition, Salvador Zubirán, Mexico City, Mexico
}

\begin{abstract}
Tactile agnosia has been described after lesions of the primary sensory cortex but the exact location and extension of those lesions is not clear.

We report the clinical features and imaging findings in a patient with an acute ischemic stroke restricted to the primary sensory area (S1). A 73-year-old man had a sudden onset of a left alien hand, without left hemiparesis. Neurological examination showed intact primary sensory functions, but impaired recognition of shape, size (macrogeometrical) and texture (microgeometrical) of objects; damage confined to the post-central gyrus, sparing the posterior parietal cortex was demonstrated on MRI. An embolic occlusion of the anterior parietal artery was suspected as mechanism of stroke.

Tactile agnosia with impaired microgeometrical and macrogeometrical features' recognition can result from a single lesion in the primary sensory cortex (S1) in the right parietal hemisphere, sparing other regions of the cerebral cortex which presumably participate in tactile object recognition.
\end{abstract}

Key words: Sensory cortex, stroke, tactile agnosia.

\section{Introduction}

Although much has been written on the subject of tactile agnosia since the time of the classical description by Dejerine, ("syndrome sensitif cortical"), ${ }^{[1,2]}$ it is not clear what is the exact nature of the clinical deficit when the lesion is confined to the post-central gyrus or primary sensory area. In this regard it has been difficult also to decide what signs may be attributed to the damage of the primary sensory area (Brodmann areas 1,2 and 3) and what signs may be attributed to the posterior parietal areas (Brodmann areas 5 and 7). The present case affords a unique opportunity to evaluate the effects of damage to the primary sensory area.

\section{Case Report}

A 73-year-old man had the sudden onset of the sensation that the left hand did not belong to him. He also noticed difficulty in recognizing objects with that hand. There was no weakness of the face, the left upper or lower extremity.

He was examined $48 \mathrm{~h}$ after the onset of symptoms. He had no hemianopia, limb weakness, hyper-reflexia of the left upper extremities and no Babinski sign. The sensory examination of the hand was as follows: Pinprick sensation was equal in both hands; cold and warm sensations were normal with water tubes at 20 and 37 degrees Celsius respectively. Two points discrimination was $1 \mathrm{~mm}$ in the tips of the fingers of both hands; vibratory sensation was found to be equal in both hands, threshold sensitivity for vibration in the left hand was $2 / 50$, and in the right hand was $2 / 50$ (BIOTHESIOMETER Biomedical Instruments Co, Newbury, Ohio), considering a normal threshold of 2/50 to $4 / 50$. Position sense was normal in the distal and proximal joints of the fingers; barognosis was equal in both hands. There was decreased recognition of numbers and letters written in the palm of the left hand (17 out of 20 times could not recognize a number or a letter). There was decreased recognition of the shape, size (macrogeometrical) and texture (microgeometrical) of an object placed on the palm of the left hand and rolled over the fingers (7 out 10), could not recognize the object placed in the palm of the left hand and examine it with the fingers either passively or actively (13 out 15). There was extinction with bilateral simultaneous stimulation at the tip of the fingers on the left hand 
$50 \%$ of times. There was a marked difficulty in bringing an object placed in the palm of the left hand into the tip of the fingers with the eyes closed (tactile apraxia). There was also a marked difficulty recognizing the direction of a tip of a pencil running in the palm of the left hand or in the left fingers; all these clinical deficits were not found on the right side. Decreased amplitude of the cortical somatosensory evoked potential with left median nerve stimulation was found on the right side. There was absence of the long latency responses in the abductor pollicis brevis on the affected side with stimulation of the median nerve at the wrist. We reexamined the patient one and six months later and found identical signs.

A brain MRI was performed $48 \mathrm{~h}$ after the onset of symptoms, showing ischemic damage confined to the post-central gyrus and a small lesion in the prefrontal cortex [Figure 1]. The main lesion was confined to the areas 1, 2 and 3 of Brodmann or S1 and spared the posterior parietal cortex areas 5 and 7 , secondary sensory cortex (S2), and the medial part of S1 cortex.

The patient was a physician who had training in internal medicine and psychiatry. He had no history of arterial hypertension, diabetes, dyslipidemia, stroke or transient ischemic attacks, but had a history of intense cigarette smoking and alcohol abuse. Atrial fibrillation was excluded with ECG and 72-h cardiac rhythm monitoring. US showed non-ulcerated bilateral atheromas at the origin of both internal carotid arteries with stenosis less than $50 \%$ by NASCET. No further studies were performed because the patient refused. He died a year later of pneumonia and no autopsy was performed.

\section{Discussion}

The main findings in this case are related to the clinical deficits that result in lesions restricted to the primary sensory cortex (S1). Recently, some authors have pointed out that the underlying failure in tactile agnosia is mainly impaired perception of microgeometrical properties (such as textures) of objects due to lesions in the primary sensory cortex (SI) and that macrogeometrical properties (such as shape and size) are recognized in the secondary somatosensory area (SII) and the posterior parietal cortex in a parallel processing fashion. ${ }^{[3,4]}$ However, our patient had problems with the recognition of both properties (micro and macrogeometrical) of objects with a lesion restricted to SI which suggests that at least in some individuals all these functions could be impaired with a single lesion in the primary sensory cortex.

It has been suggested that a right hemisphere lesion with sparing of right SI cortex produces a deficit in exploratory motor procedures that results in a pure tactile apraxia without agnosia, which can produce a deficit in tactile object recognition (TOR) when

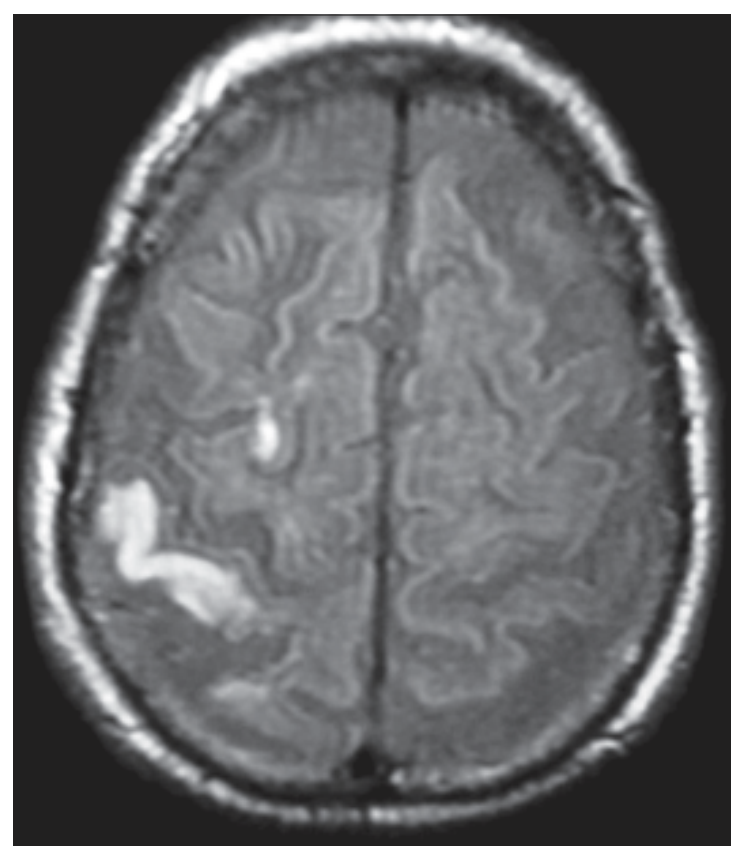

Figure 1: FLAIR axial section of the brain showing a hyperintense lesion confined to the post-central gyrus on the right hemisphere. There is a small lesion in the prefrontal cortex

the patient explores objects passively (spontaneous exploration) and not actively (manually or verbally guided exploration) ${ }^{[5]}$ Interestingly, our case presented features of tactile apraxia, even though the lesion was confined to a single area in the post-central gyrus.

Using functional magnetic resonance imaging (MRI) in healthy subjects, it has been shown that the right superior parietal cortex is specifically activated by kinaesthetic attention during tactile object discrimination. ${ }^{[6]}$ Also, functional MRI in healthy subjects has been used to demonstrate that TOR involves the calcarine cortex, inferior parietal lobule, inferior frontal gyrus and superior frontal gyrus polar region, and may utilize visual systems to access an internal object representation with a lexical strategy of naming the objects being examined. ${ }^{[7]}$ One possible explanation for the topography of the stroke is an occlusion of the anterior parietal artery, which is usually a branch of the inferior division of the middle cerebral artery. We suspected artery-artery embolism from the right carotid plaque as mechanism for arterial occlusion.

\section{References}

1. Dejerine J. Sémiologie des affections du systéme nerveux. Masson Editeurs 1914. p. 915-8.

2. Caselli RJ. Rediscovering tactile agnosia. Mayo Clin Proc 1991;66:129-42.

3. Bohlhalter S, Fretz C, Weder B. Hierarchical versus parallel processing in tactile object recognition: A behavioural-neuroanatomical study of aperceptive tactile agnosia. Brain 2002;125:2537-48.

4. Burton H, Sinclair R.J. Attending to and remembering tactile stimuli: A review of brain imaging data and single-neuron responses. J Clin Neurophysiol 2000;17:575-91. 
5. Valenza N, Ptak R, Zimine I, Badan M, Lazeyras F, Schnider A. Dissociated active and passive tactile shape recognition: A case study of pure tactile apraxia. Brain 2001;124:2287-98.

6. Stoeckel MC, Weder B, Binkofski F, Choi HJ, Amunts K, Pieperhoff P, et al. Left and right superior parietal lobule in tactile object discrimination. Eur J Neurosci 2004;19:1067-72.
7. Deibert E, Kraut M, Kremen S, Hart J Jr. Neural pathways in tactile object recognition. Neurology 1999;52:1413-7.

Accepted on 05-10-2008

Source of Support: Nil, Conflict of Interest: None declared.

\section{Author Help: Sending a revised article}

1) Include the referees' remarks and point to point clarification to those remarks at the beginning in the revised article file itself. In addition, mark the changes as underlined or coloured text in the article. Please include in a single file

a. referees' comments

b. point to point clarifications on the comments

c. revised article with text highlighting the changes done

2) Include the original comments of the reviewers/editor with point to point reply at the beginning of the article in the 'Article File'. To ensure that the reviewer can assess the revised paper in timely fashion, please reply to the comments of the referees/editors in the following manner.

- There is no data on follow-up of these patients.

Authors' Reply: The follow up of patients have been included in the results section [Page 3, para 2]

- $\quad$ Authors should highlight the relation of complication to duration of diabetes.

Authors' Reply: The complications as seen in our study group has been included in the results section [Page 4, Table] 